\title{
Lipidomics of Alzheimer's disease: a liver peroxisomal dysfunction in the metabolism of omega- 3 fatty acids
}

\author{
Giuseppe ASTARITA \\ Daniele PIOMELLI
}

University of California Irvine, Department of Pharmacology, University of California, Irvine, $C A$ 92697-4625, (G.A., D.P.),

Department of Biological Chemistry, University of California, Irvine, CA 92697-4625 (D.P.), United States Unit of Drug Discovery and Development, Italian Institute of Technology, Genoa, Italy (G.A., D.P.)

\begin{abstract}
Reduced brain levels of docosahexaenoic acid (DHA), a neurotrophic and neuroprotective fatty acid, might contribute to cognitive and visual decline. A multiorgan lipidomic approach revealed that the levels of free DHA, DHA-containing phospholipids precursors and DHA-metabolites were decreased in various brain regions of subjects with Alzheimer's disease (AD), compared to control subjects. Furthermore, liver DHA content was lower in AD patients than control subjects. Shorter chain omega-3 fatty acids precursors for DHA, were elevated in liver of AD patients, whereas expression of peroxisomal D-bifunctional protein, which catalyzes the conversion of tetracosahexaenoic acid into DHA, was selectively reduced. Lower DHA levels in the liver were associated with cognitive impairment and with higher prevalence of eye-diseases. The evidence presented herein indicate that a deficit in liver biosynthesis of DHA possibly lessens the flux of this neuroprotective fatty acid to brain and eyes, contributing to cognitive and visual impairments.
\end{abstract}

Key words : DHA, omega-3 fatty acids, liver, Alzheimer's disease, cognition, eyes
Emerging epidemiological and molecular evidence indicates that a variety of lipid abnormalities are associated with Alzheimer's disease (AD) and, possibly, involved in its pathogenesis (Cunnane et al., 2009). Risk factors for AD - such as aging, genetic vulnerability and environmental factors - alter specific lipid pathways in brain and peripheral tissues, and these alterations may influence in turn AD progression (figure 1). For example, the inheritance of certain forms of the lipid-carrier protein apolipoprotein $\mathrm{E}$ ( $A P O E$ ) increases the risk of $A D$ and brain tissue from $A D$ patients shows significant changes in lipid composition. Among the alterations most consistently observed in $A D$ are decreases in levels of docosahexaenoic acid (C22:6, DHA), an omega-3 fatty acid derived from diet or synthesized in the liver. Although a large number of pre-clinical and clinical evidence suggests that DHA decreases the risk of developing $A D$, it is still unclear how $A D$ affects the metabolism of this essential omega-3 fatty acid.

Further understanding of the DHA metabolism in a complex disorder such as $A D$ requires a more integrated lipidomics strategy, which can be regarded at three different levels (figure 2). First, from the molecular point of view, putting together lipidomics information with genomic, transcriptomic, proteomics and metabolomics data would give access to a global view of molecular changes underlying age-related cognitive decline and $\mathrm{AD}$ (figure 2). The second level of integration is from a more functional perspective, taking into account the demographic characteristics and clinical information of the subjects under study (figure 2). This level of information would allow relating the lipid changes with the initiation and progression of cognitive decline and AD. The third possible level of integration is from a more holistic prospect, taking under consideration the interaction between peripheral organs and brain (figure 2). Indeed, most of the brain DHA derive from peripheral tissues through either biosynthetic processes (e.g., in liver) or dietary absorption. Consequently, systemic changes in lipid metabolism occurring in the peripheral tissues might strongly affect the structure and functioning of the brain. Only few studies have dealt with multi-organ interaction in $A D$ and, at the moment, the role of peripheral lipid metabolism during cognitive decline and $A D$ development is still unclear.

Together, this integrative holistic approach, which could be called "Systems Lipidomics" (figure 2), could open a new dimension for both differential and functional analyses of multifactorial disorders. In the present review, we analyze the application of a multi-organ

To cite this article: Astarita G, Piomelli D. Lipidomics of Alzheimer's disease: a liver peroxisomal dysfunction in the metabolism of omega-3 fatty acids. OCL 2011;18(4):218-23. doi : 10.1684/ocl.2011.0387 


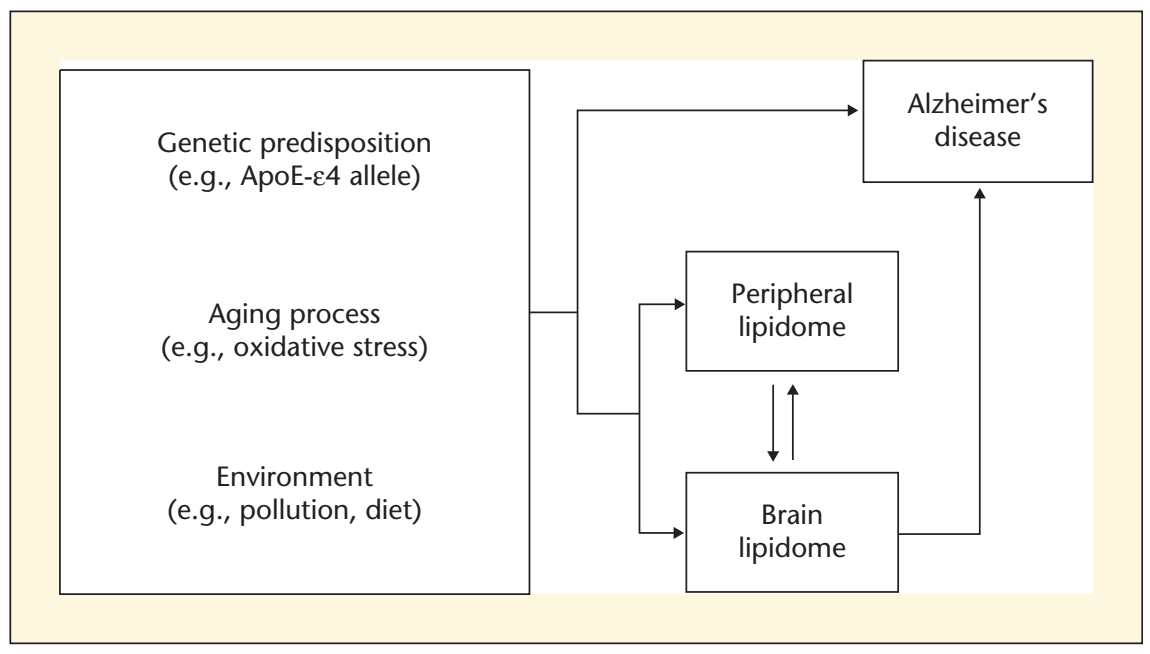

Figure 1. Scheme illustrating the multifactorial etiology of $A D$. Risk factors for $A D$ (which include genetic-predisposition, aging and environmental factors such as a nutritional deficit in omega-3 fatty acids) influence interacting lipidomes throughout the body. Over time, accumulating lipid changes compound with those factors to increase the risk for $A D$.

Systems Lipidomics approach to study the metabolism of omega-3 fatty acids in $A D$.

\section{Free DHA levels in AD brain}

With some inconsistency, deficits in DHAcontaining phospholipids have been

reported in AD brains (Cunnane et al., 2009). Only one previous study reported the levels of free DHA to be lower in hippocampus of AD patients than control subjects (Lukiw et al., 2005). We measured the brain levels of free DHA by liquid chromatography/mass spectrometry (LC/MS) from mid-frontal cortex samples of 17 control subjects and $37 \mathrm{AD}$ patients, which were matched for age

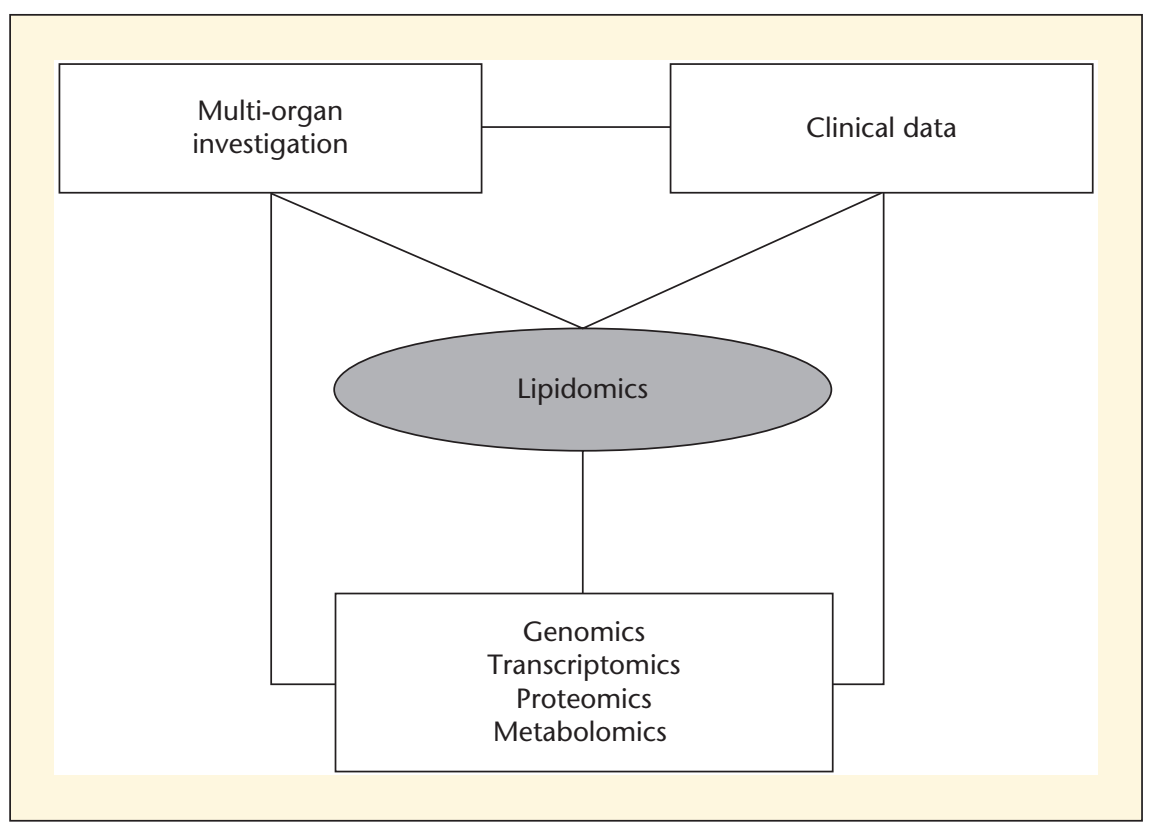

Figure 2. Systems Lipidomics for descriptive and functional analysis of complex disorders. Integration at both molecular and physiopathological levels is required for the understanding of complex diseases. It is to be noted that this schematic representation applies not only to $A D$, but to most human pathologies, and can be used for the identification and validation of novel biomarkers and therapeutic targets. and post-mortem interval. DHA levels were significantly lower $(\mathrm{P}<0.05)$ in midfrontal cortex of AD patients compared to those of control subjects (figure $3 A$ ) (Astarita et al., 2010).

\section{DHA-containing Phospholipids in AD brains}

Free DHA is in equilibrium with DHA-containing glycerophospholipids (figure 4), which could be hydrolyzed into free DHA by phospholipases $A_{2}$. The levels of the 1-stearoyl, 2-docosahexaenoyl-sn-glycero-3-phosphoethanolamine were significantly lower in mid-frontal cortex of AD patients than control subjects (figure $3 B$ ).

\section{DHA metabolites in AD brains}

Free DHA may be converted by a lipoxygenase-mediated pathway into neuroprotectin D1 (NPD1) (figure 4), a lipid signal with marked anti-inflammatory and neuroprotective properties (Hong et al., 2003). Previous work by Lukiw et al. (2005) reported NPD1 levels are decreased in temporal lobes of subjects with AD. In our study, we observed that NPD1 levels were strikingly decreased in mid-frontal cortex of subjects with $A D$ compared to control subjects ( $P=0.0018)$. Because NPD1 has been implicated in the survival and repair of neuronal cells, such deficit could contribute to the brain damage and neural death observed in AD brains.

\section{DHA levels in AD livers}

Liver's capacity to generate DHA is critical to keep the bioavailability of this fatty acid within a normal range, especially when food does not provide a sufficient supply of this nutrient (Rapoport et al., 2007; Rapoport et al., 2007). A properly functioning liver synthesizes DHA from shorter-chain omega-3 precursors, such as $\alpha$-linolenic acid (ALA, C18:3, omega3) and eicosapentaenoic acid (C20:5, omega-3) (Rapoport et al., 2007; Scott and Bazan, 1989; Burdge and Calder, 2005) (figure 5). A cascade of elongase and desaturase enzymes localized in the endoplasmic reticulum of the hepatocyte progressively add carbon units and 


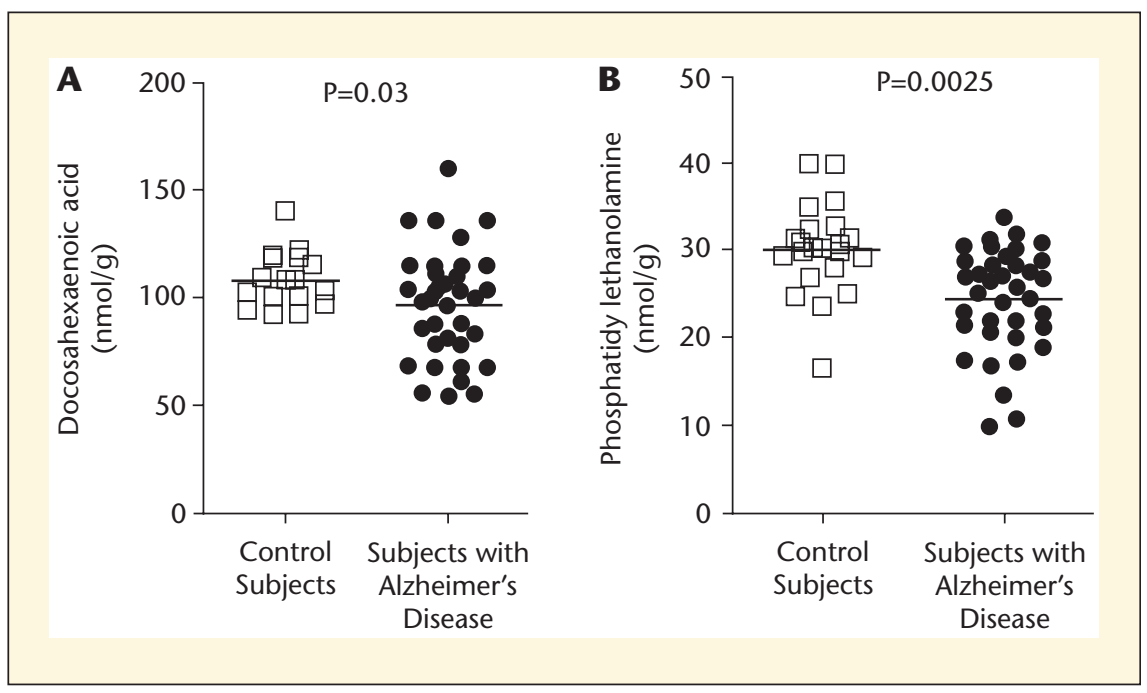

Figure 3. DHA content in AD brain. Levels of DHA (A) and DHA-containing phosphatidylethanolamine (1-stearoyl,2-arachidonoyl-3-glycerophosphoethanolamine, B) in mid-frontal cortex of control subjects (open squares) and subjects with $A D$ (closed circles). Statistical analyses were conducted using a linear regression analysis adjusting for age, gender and post mortem interval.

double bonds to shorter-chain omega-3 fatty acids, producing the very-longchain tetracosahexaenoic acid (C24:6, omega-3). This fatty acid is transported into peroxisomes and then converted to DHA by the sequential action of acylcoenzyme A oxidases, D-bifunctional protein (DBP) and peroxisomal thiolases (Voss et al., 1991, Sprecher et al., 1995, Moore et al., 1995, Su et al., 2001) (figure 5). Liver-derived DHA reaches the

eyes and brain through the general circulation, probably bound to lipoprotein transporters, such as albumin (Scott and Bazan, 1989) (figure 4). Consequently, changes in DHA metabolism in the liver might strongly affect the structure and functioning of tissues that heavily rely on DHA bioavailability (i.e., the eyes and the brain).

Current evidence indicates that the livermediated DHA biosynthesis may be suffi-

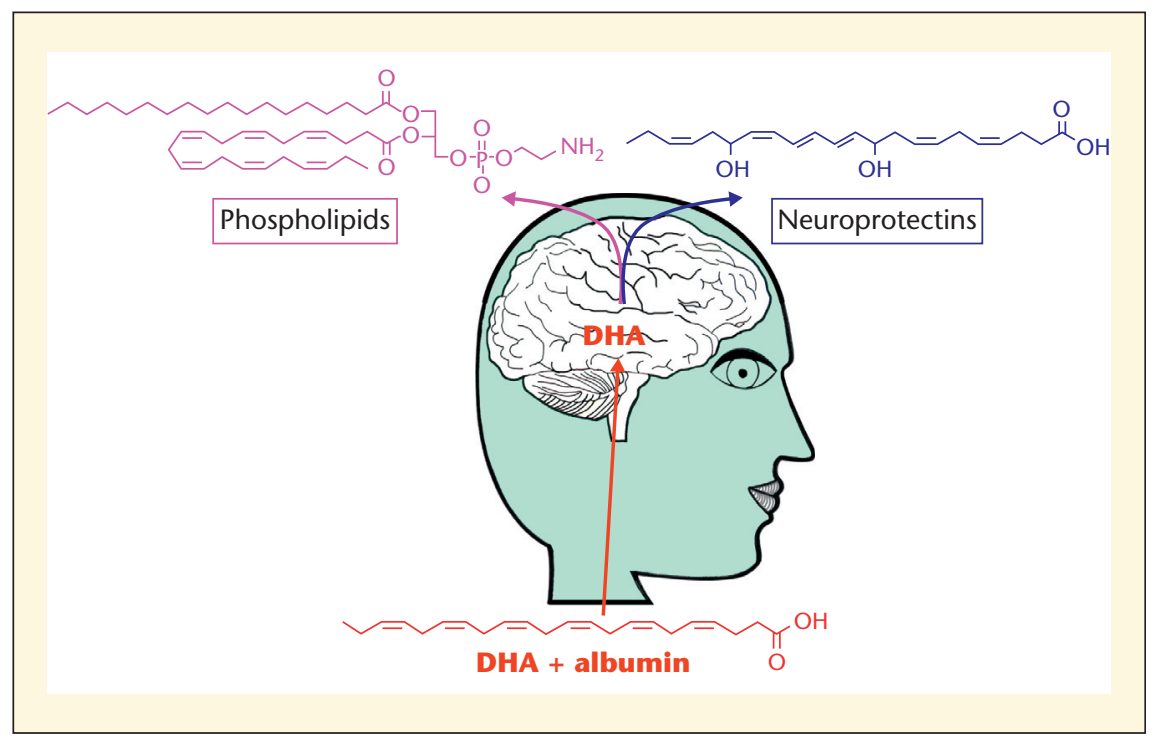

Figure 4. DHA transport through the blood to eye and brain tissues Liver-derived DHA reaches the eyes and the brain (two tissues highly enriched in DHA) through the general circulation, most likely as a complex with lipid-binding proteins (e.g., albumin) that are also synthesized in the hepatocytes. Once in the brain, free DHA is in equilibrium with complex lipids (e.g., phosphatidylethanolamines) and metabolites (e.g., neuroprotectins). The figure shows chemical structures of DHA-derived lipids quantified in our analyses. cient (and indeed essential) for human DHA requirements (Rapoport and Igarashi, 2009; Scott and Bazan, 1989). Indeed, human brain consumes DHA at the incredible slow rate of $3.8 \mathrm{mg} /$ day (Umhau et al., 2009). Considering that $0.5-10 \%$ of ingested ALA is converted in $\mathrm{DHA}$, and an average ingestion of $1,400 \mathrm{mg} /$ day of ALA (Albert et al., 2005), the liver is able to synthesize DHA at rates of 7-140 mg/day, 1.8-36fold, respectively, the human brain requirement (Umhau et al., 2009). It appears, however, that an overall healthy liver is required for optimal DHA biosynthesis. Indeed, it has been reported that during conditions of hepatic stress such as in chronic alcohol intake (Pawlosky and Salem Jr, 1999, Frye and Salloum, 2006), and liver steatosis and injury (Araya et al., 2004, Allard et al., 2008), the levels of hepatic DHA are compromised.

To examine whether the metabolic conversion of ALA into DHA is altered in $A D$, we recently quantified the omega-3 fatty acids in liver samples from a cohort of 9 control subjects and 14 AD patients (Astarita et al., 2010). $D H A$ levels were lower in liver of $A D$ patients compared with control subjects (table 1). The levels of the shorter chain omega- 3 fatty acids that function as precursors for DHA were increased in liver of $A D$ patients compared with control subjects (table 1). These results suggested that a dysfunction in the biosynthesis of DHA might be involved in the deficit of this essential fatty acid.

\section{DHA biosynthesis in liver}

To better understand the molecular step that may be dysregulated in the liver omega-3 metabolism, we performed mRNA analyses of targeted genes involved in the biosynthesis of DHA (table 2). The targeted analysis included both cytosolic enzymes, which are involved in the elongation and desaturation steps, and peroxisomal enzymes, which are involved in the peroxisomal biogenesis, fatty acid transport, and fatty acid beta-oxidation. The mRNA encoding the peroxisomal enzyme D-bifunctional protein (DBP, hydroxysteroid (17beta) dehydrogenase 4, HSD17B4) was lower in the livers of $A D$ patients than control subjects (table 2). No changes were observed for the other enzymes. Our results are in agreement with the reports showing a decline in the 


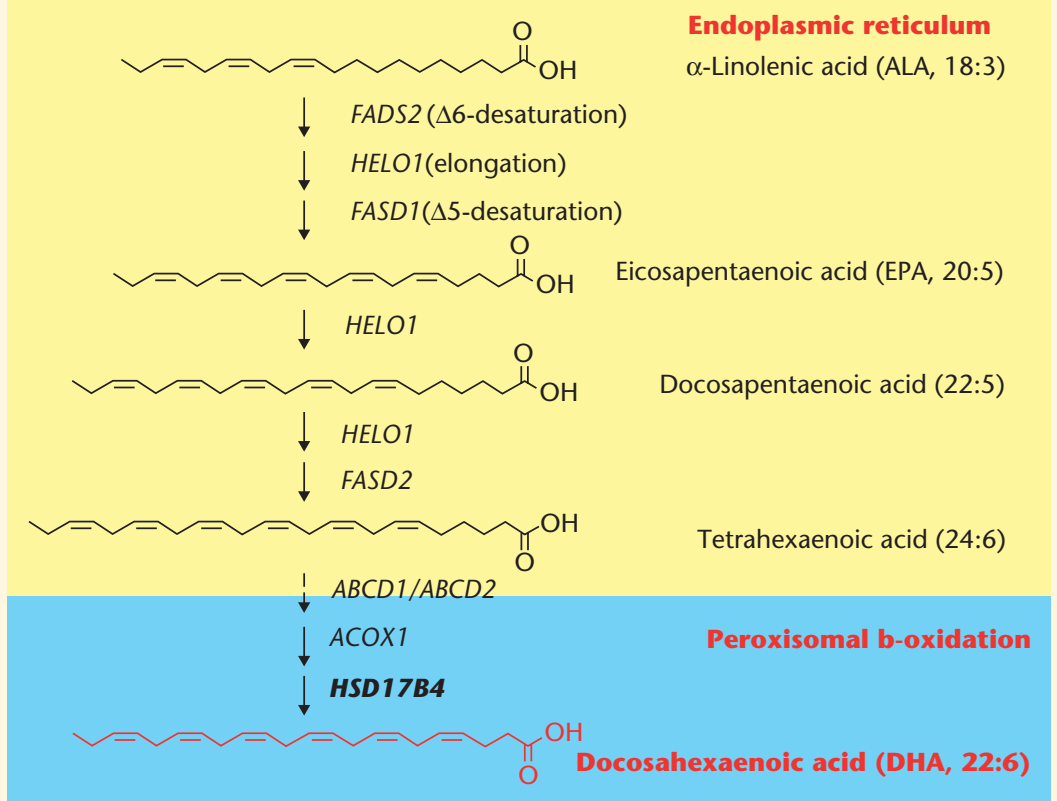

Figure 5. Overview of DHA biosynthesis in liver. Diet-derived ALA (C18:3 omega-3) is transformed into tetracosahexaenoic acid (C24:6 omega-3) by the sequential action of $\Delta 6$ and $\Delta 5$ desaturases (encoded by the FADS2 and FADS1 genes, respectively) and elongases (such as that encoded by the HELO1 gene) present in the endoplasmatic reticulum. Tetracosahexaenoic acid is transported into peroxisomes (shaded area), presumably by proteins encoded by the $\mathrm{ABCD} 1$ or $\mathrm{ABCD} 2$ genes, and then converted into docosahexaenoic acid (DHA, C22:6 omega3 ) by sequential action of acyl coenzyme-A oxidase (encoded by the ACOX1 gene), Dbifunctional protein (encoded by the HSD17B4 gene), and various peroxisomal thiolases (not shown). The figure shows chemical structures of fatty acids quantified in our analyses.

peroxisomal function associated with aging (Youssef and Badr, 1999). A peroxisomal dysfunction could also explain the decrease in the synthesis of both plasmalogens (Zoeller and Raetz, 1986; Martinez, 1990; Favrelere et al., 2000) and DHA (Burdge and Calder, 2005; Burdge et al., 2003; Burdge et al., 2002) observed in elderly people. Overall, this evidence supports the hypothesis that peroxisomal DHA biosynthesis may have a significant role in aging and AD.

\section{Liver DHA is linked to cognitive impairment}

Several, albeit not all, epidemiological and clinical studies suggest that higher intake of DHA decreases the risk of cognitive decline and dementia in elderly adults. Notably, our study revealed that liver DHA correlated with the most recent MMSE and global deterioration scale scores. The ratios were positively correlated with MMSE scores ( $r=0.78$;
$\mathrm{P}<0.0001)$ and negatively correlated with global deterioration scale grades $(r=0.48 ; P=0.004)$. Although the increased availability of DHA might directly affect the brain lipid composition and functioning, it is likely, however, that the positive effects excited by DHA are the result of multiple signaling events, many of which remain to be discovered. For example, DHA is known to play important roles in the cardiovascular system, which in turn may contribute to the clinical manifestation and the pathology of $A D$ in the brain (Milionis et al., 2008; Beach et al., 2007). The roles of DHA on the vascular component and the cerebral parenchyma itself, however, have not been systematically explored (de la Torre, 2004).

\section{Liver DHA is linked to eye diseases}

DHA plays an essential role in eye health. Very high levels of DHA are present in the retina, accounting for over half the total fatty acyl groups present in the phospholipids of rod outer segment membranes. DHA turnover in the retina is surprisingly slow and high levels of DHA appear to be maintained despite reductions in dietary intakes of omega- 3 fatty acids. In this context, a blood stream "long loop" connect the supply of DHA to the biogenesis of excitable and photoreceptor membranes (Bazan, 1990). In retinal degenerative diseases, a shortage of blood DHA has been demonstrated, and a failure of the "long loop" from the liver is suggested to underlie these changes (Bazan et al., 1986). Notably, the retina of patients with peroxisomal disorders has virtually no DHA (Martinez, 1992) and visual improvement has been obtained in these patients using DHA therapy (Noguer and Martinez, 2009).

Table 1. Levels of free omega-3 fatty acids (nmol/g) in liver of control subjects and subjects with $A D$.

\begin{tabular}{|lccc|}
\hline \multirow{2}{*}{ Omega-3 Fatty acid } & Control subjects & Subjects with AD & P-value \\
\cline { 2 - 5 } & Mean \pm SD; $\mathbf{~ = 9}$ & Mean \pm SD; $\mathbf{~}=\mathbf{1 4}$ & \\
\hline ALA (C18:3) & $28.55 \pm 7.88$ & $36.72 \pm 22.05$ & 0.152 \\
\hline Eicosapentaenoic (C20:5) & $44.57 \pm 14.74$ & $67.4 \pm 29.46$ & 0.012 \\
\hline Docosapentaenoic (C22:5) & $21.51 \pm 8.97$ & $32.39 \pm 13.82$ & 0.019 \\
\hline Tetrahexaenoic (C24:6) & $0.73 \pm 0.17$ & $0.9 \pm 0.21$ & 0.041 \\
\hline DHA (C22:6) & $324.83 \pm 122.89$ & $204.64 \pm 74.62$ & 0.011 \\
\hline
\end{tabular}

Statistical analyses were conducted using a linear regression analysis adjusting for age, gender and post mortem interval. 
Table 2. Expression of genes involved in DHA biosynthesis and peroxisomal function in liver of control subjects and subjects with $A D$.

\begin{tabular}{|lccc|}
\hline Gene & Control Subjects & Subjects with AD & P-value \\
\hline Symbol & Mean \pm SD; $\mathbf{~}=\mathbf{9}$ & Mean \pm SD; $\mathbf{~}=\mathbf{1 4}$ & 0.851 \\
\hline FADS2 & $0.02 \pm 0.029$ & $0.016 \pm 0.0099$ & 0.265 \\
\hline HELO1 & $0.011 \pm 0.012$ & $0.006 \pm 0.0046$ & 0.693 \\
\hline FADS1 & $0.037 \pm 0.061$ & $0.024 \pm 0.026$ & 0.500 \\
\hline ABCD1 & $0.027 \pm 0.034$ & $0.02 \pm 0.024$ & 0.567 \\
\hline ABCD2 & $8 \mathrm{e}-04 \pm 0.001$ & $0.0011 \pm 0.0013$ & 0.782 \\
\hline ACOX1 & $0.2 \pm 0.24$ & $0.31 \pm 0.68$ & 0.048 \\
\hline HSD17B4 & $0.18 \pm 0.045$ & $0.14 \pm 0.046$ & 0.569 \\
\hline PEX13 & $0.041 \pm 0.017$ & $0.037 \pm 0.017$ & 0.700 \\
\hline PEX14 & $0.0087 \pm 0.004$ & $0.013 \pm 0.022$ & 0.291 \\
\hline PEX19 & $0.018 \pm 0.018$ & $0.012 \pm 0.0054$ & \\
\hline
\end{tabular}

Statistical analyses were conducted using a linear regression analysis adjusting for age, gender and RNA integrity number.

Increasing evidence shows that, besides the brain, also the eyes, and particularly the retina, are affected in $\mathrm{AD}$ (Guo et al., 2010; Hodge et al., 2005). In our population, the prevalence of eye-diseases (i.e., a combination of cataract, glaucoma and macular degeneration) was $78.5 \%$ in AD patients versus 33.5\% in control subjects. Lower DHA levels in the liver were associated with higher prevalence of eye-diseases $(P=0.0016)$, indicating a previously unrecognized association between hepatic DHA availability and eye-health. These results emphasize the potential role of liverderived DHA in visual health.

\section{Conclusion}

The use of a multi-organ systems lipidomics approach is contributing to our understanding of the regulation of DHA metabolism in AD. It appears that a reduced DHA bioavailability - due to a dysfunctional liver biosynthesis - leads to a series of molecular and functional changes that might contribute to the cognitive and visual impairments observed in AD patients. Further investigation on the role of the hepatic peroxisomal metabolism in brain and eye health is currently underway.

\section{REFERENCES}

Albert CM, Oh K, Whang W, et al. Dietary alpha-linolenic acid intake and risk of sudden cardiac death and coronary heart disease. Circulation 2005; 112: 3232-8.
Allard JP, Aghdassi E, Mohammed S, et al. Nutritional assessment and hepatic fatty acid composition in non-alcoholic fatty liver disease (NAFLD): a cross-sectional study. I Hepatol 2008; 48: 300-7.

Araya J, Rodrigo R, Videla LA, et al. Increase in long-chain polyunsaturated fatty acid $n-6 / n$ - 3 ratio in relation to hepatic steatosis in patients with non-alcoholic fatty liver disease. Clin Sci (Lond) 2004; 106: 635-43.

Astarita G, Jung K, Berchtold $\mathrm{N}$, et al. Deficient liver biosynthesis of docosahexaenoic acid correlates with cognitive impairment in Alzheimer's disease. PLoSOne 2010; 5: e12538.

Bazan NG, Scott BL, Reddy TS, Pelias MZ. 1986. Decreased content of docosahexaenoate and arachidonate in plasma phospholipids in Usher's syndrome. Biochem Biophys Res Commun 141: 600-4.

Bazan N. Supply of $n-3$ polyunsaturated fatty acids and their significance in the central nervous system. In: R. Wurtman and J. Wurtman, eds. Nutrition and the Brain. Raven Press Ltd, NY, 1990: 1-24.

Beach TG, Wilson JR, Sue LI, et al. Circle of Willis atherosclerosis: association with Alzheimer's disease, neuritic plaques and neurofibrillary tangles. Acta Neuropathol 2007; 113: 13-21.

Burdge GC, Jones AE, Wootton SA. Eicosapentaenoic and docosapentaenoic acids are the principal products of alpha-linolenic acid metabolism in young men. Br J Nutr 2002; 88: 355-63.

Burdge GC, Finnegan YE, Minihane AM, Williams CM, Wootton SA. Effect of altered dietary n-3 fatty acid intake upon plasma lipid fatty acid composition, conversion of [13C] alpha-linolenic acid to longer-chain fatty acids and partitioning towards beta-oxidation in older men. Br J Nutr 2003; 90: 311-21.

Burdge G, Calder P. Conversion of alphalinolenic acid to longer-chain polyunsaturated fatty acids in human adults. Reprod Nutr Dev 2005; 45: 581-97.

Cunnane SC, Plourde M, Pifferi F, Begin M, Feart C, Barberger-Gateau P. Fish, Docosahexaenoic Acid and Alzheimer's Disease. Prog Lipid Res 2009; 48: 239-56.

de la Torre JC. Is Alzheimer's disease a neurodegenerative or a vascular disorder? Data, dogma, and dialectics. Lancet Neurol 2004; 3: 184-90.

Favrelere S, Stadelmann-Ingrand S, Huguet F, et al. Age-related changes in ethanolamine glycerophospholipid fatty acid levels in rat frontal cortex and hippocampus. Neurobiol Aging 2000; 21: 653-60.

Frye MA, Salloum IM. Bipolar disorder and comorbid alcoholism: prevalence rate and treatment considerations. Bipolar Disord 2006; 8: 677-85.

Guo L, Duggan J, Cordeiro MF. Alzheimer's disease and retinal neurodegeneration. Curr Alzheimer Res 2010; 7: 3-14.

Hodge W, Barnes D, Schachter HM, et al. Effects of omega-3 fatty acids on eye health. Evid Rep Technol Assess (Summ) 2005 : 1-6.

Hong S, Gronert K, Devchand PR, Moussignac RL, Serhan CN. Novel docosatrienes and 17 S-resolvins generated from docosahexaenoic acid in murine brain, human blood, and glial cells. I Biol Chem 2003; 278: 14677-87.

Lukiw WJ, Cui JG, Marcheselli VL, et al. A role for docosahexaenoic acid-derived neuroprotectin D1 in neural cell survival and Alzheimer disease. J Clin Invest 2005; 115: 2774-83. 
Martinez M. Severe deficiency of docosahexaenoic acid in peroxisomal disorders: a defect of delta 4 desaturation? Neurology 1990; 40: 1292-8.

Martinez M. Abnormal profiles of polyunsaturated fatty acids in the brain, liver, kidney and retina of patients with peroxisomal disorders. Brain Res 1992; 583: 171-82.

Milionis HJ, Florentin M, Giannopoulos S. Metabolic syndrome and Alzheimer's disease: a link to a vascular hypothesis? CNS Spectr 2008; 13: 606-13.

Moore SA, Hurt E, Yoder E, Sprecher $H$, Spector AA. Docosahexaenoic acid synthesis in human skin fibroblasts involves peroxisomal retroconversion of tetracosahexaenoic acid. J Lipid Res 1995; 36: 2433-43.

Noguer MT, Martinez M. Visual follow-up in peroxisomal-disorder patients treated with docosahexaenoic acid ethyl ester. Invest Ophthalmol Vis Sci 2009; 51: 2277-85.

Pawlosky RJ, Salem Jr N. Alcohol consumption in rhesus monkeys depletes tissues of polyunsaturated fatty acids and alters essential fatty acid metabolism. Alcohol Clin Exp Res 1999; 23: 311-7.

Rapoport S, Rao J, Igarashi M. Brain metabolism of nutritionally essential polyunsaturated fatty acids depends on both the diet and the liver. Prostaglandins Leukot Essent Fatty Acids 2007; 77: 251-261.

Rapoport SI, Igarashi M. Can the rat liver maintain normal brain DHA metabolism in the absence of dietary DHA? Prostaglandins Leukot Essent Fatty Acids 2009; 81: 119-23.

Scott B, Bazan N. Membrane docosahexaenoate is supplied to the developing brain and retina by the liver. Proc Natl Acad Sci U S A 1989; 86: 2903-7.

Sprecher H, Luthria DL, Mohammed BS, Baykousheva SP. Reevaluation of the pathways for the biosynthesis of polyunsaturated fatty acids. J Lipid Res 1995; 36: 2471-7.

Su HM, Moser AB, Moser HW, Watkins PA. Peroxisomal straight-chain Acyl-CoA oxidase and D-bifunctional protein are essential for the retroconversion step in docosahexaenoic acid synthesis. J Biol Chem 2001; 276: 3811520.

Umhau JC, Zhou W, Carson RE, et al. Imaging incorporation of circulating docosahexaenoic acid into the human brain using positron emission tomography. J Lipid Res 2009; 50: 1259-68.

Voss A, Reinhart M, Sankarappa S, Sprecher $\mathrm{H}$. The metabolism of $7,10,13,16,19$-docosapentaenoic acid to 4,7,10,13,16,19-docosahexaenoic acid in rat liver is independent of a 4-desaturase. J Biol Chem 1991; 266: 19995-20000.

Youssef J, Badr M. Biology of senescent liver peroxisomes: role in hepatocellular aging and disease. Environ Health Perspect 1999; 107: 791-7.

Zoeller RA, Raetz CR. Isolation of animal cell mutants deficient in plasmalogen biosynthesis and peroxisome assembly. Proc Natl Acad Sci U S A 1986; 83: 5170-4. 